\title{
Self-annihilation of antiphase boundaries in GaAs epilayers on Ge substrates grown by metal-organic vapor-phase epitaxy
}

\author{
M. K. Hudait ${ }^{\text {a) }}$ and S. B. Krupanidhi \\ Materials Research Centre, Indian Institute of Science, Bangalore-560 012, India
}

\begin{abstract}
The self-annihilation of antiphase boundaries (APBs) in GaAs epitaxial layers grown by low-pressure metal-organic vapor-phase epitaxy on $\mathrm{Ge}$ substrates is studied by several characterization techniques. Cross-sectional transmission electron microscopy shows that antiphase domain free GaAs growth on Ge was possible due to the proper selection of the growth parameters. The antiphase boundaries annihilate with each other after a thick $3 \mu \mathrm{m}$ layer of GaAs growth on a Ge substrate as observed by scanning electron microscopy studies. Double crystal x-ray diffraction data shows a slight compression of GaAs on $\mathrm{Ge}$, and the full width at half maximum decreases with increasing growth temperatures. This confirms that the APBs annihilate inside the GaAs epitaxial films. Low temperature photoluminescence measurements confirm the self-annihilation of the APBs at low temperature growth and the generation of APBs at higher growth temperatures.
\end{abstract}

\section{INTRODUCTION}

$\mathrm{GaAs} / \mathrm{Ge}$ heterostructures have received a great deal of attention from the space solar cells and the electronics and optoelectronics communities. Due to its high mechanical strength, Ge is an optimized substrate material in terms of its power-to-weight ratio for high efficiency GaAs/Ge solar cells. As large area, minority carrier devices, III-V on Ge cells is extremely sensitive to defects. Thus elimination of antiphase domains (APDs), which are characteristics of polar-on-nonpolar epitaxy, and the suppression of large-scale interdiffusion across the GaAs/Ge heterointerface remain key challenges for increased yield, reliability, and performance of the devices.

The low lattice mismatch of the GaAs/Ge system $(0.07 \%$ at room temperature and $0.12 \%$ at growth temperature) suggests that it should be nearly dislocation free. However, polar-on-nonpolar heteroepitaxy poses several unique problems of its own, like APDs, Ge outdiffusion into the GaAs epitaxial film, and unwanted $p-n$ junction formation by simultaneous in diffusion of $\mathrm{Ga}$ and As into the Ge substrate. The formation of APDs is associated with all polar-onnonpolar heteroepitaxy. The reason is that when polar materials (e.g., III-V compounds) are epitaxially grown on nonpolar substrates (e.g., $\mathrm{Ge}$ or $\mathrm{Si}$ ), the location of cation atoms and anion atoms in the two sublattices can reverse from area to area in the epilayer, ${ }^{1}$ which forms APDs. Domains of different sublattice locations are separated by an antiphase boundary (APB) consisting of wrong bonds of As-As or $\mathrm{Ga}-\mathrm{Ga}$. APBs are expected give rise to deep levels inside the forbidden band and to act as strong scattering centers for electrons and holes. ${ }^{1-3}$

\footnotetext{
a) Author to whom correspondence should be addressed; present address: Department of Electrical Engineering, The Ohio State University, 2015 Neil Avenue, Columbus, OH 43210-1272; electronic mail: hudaitm@ee.eng.ohio-state.edu
}

Apart from the different models concerning the suppression of the formation of APDs during nucleation, ${ }^{1,2,4}$ selfannihilation of APBs is reported to play an important role in APB-free heteroepitaxy of GaAs on $\mathrm{Ge}$ and $\mathrm{Si}$ (100) substrates. ${ }^{5-25}$ Kawabe and $\mathrm{Ueda}^{6}$ and Fischer et al. ${ }^{7}$ have reported the presence of the self-annihilation process of APBs during molecular beam epitaxy (MBE) growth of GaAs on $\mathrm{Si}$ (001), as evidenced by a transition of the highenergy electron diffraction pattern from a mixed $(2 \times 4)$ GaAs surface to a resolved $(2 \times 4)$ surface. To explain this phenomenon a model was proposed in two of their papers. $\mathrm{Li}$ and Giling ${ }^{12}$ studied the self-annihilation process of APBs in GaAs epilayers grown on Ge by metal-organic vapor-phase epitaxy (MOVPE). The three-dimensional development of APBs is recorded by growth of GaAs on a GaAs surface already containing APBs and layer-by-layer APB-revealing etching followed by mechanochemical polishing of a GaAs epilayer, which contains APBs. They suggest a new model, a $\{011\}$ APB model rather than the $\{111\}$ mechanism suggested in the literature.

In this article we will show some evidence for the selfannihilation of APBs in GaAs epilayers studied experimentally using scanning electron microscopy (SEM), highresolution transmission electron microscopy (HRTEM), double crystal x-ray diffraction (DCXRD), and photoluminescence (PL) studies. The aim of this work is not to propose concerning the structure and the properties of APBs, but to show some evidence of the annihilation of APBs during the low-pressure metal-organic vapor-phase epitaxy (LPMOVPE) growth of GaAs and Ge. This will give a clear indication of how the APBs are self-annihilated during the thick GaAs epitaxial layer.

\section{EXPERIMENT}

Undoped and Si-doped $n$-type GaAs epitaxial layers of different thicknesses were grown in a horizontal LPMOVPE 


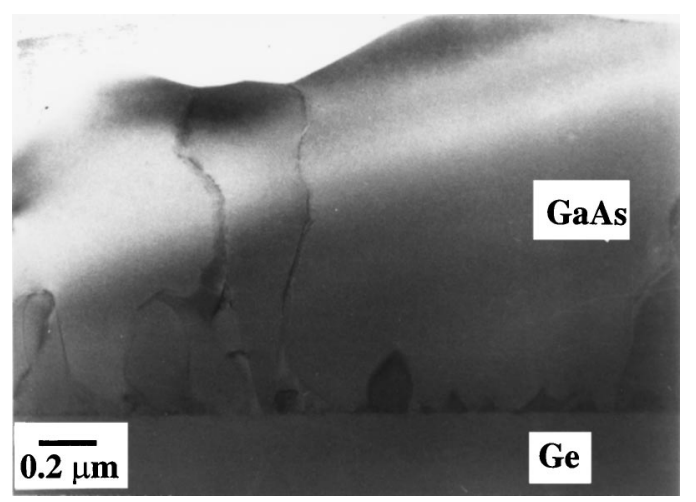

FIG. 1. [110] TEM cross-sectional micrograph of the GaAs/Ge heterointerface grown at $700{ }^{\circ} \mathrm{C}$ and a growth rate of $3 \mu \mathrm{m} / \mathrm{h}$ with a V/III ratio of 88.20 .

reactor on (100) $n^{+}$-Ge substrates off-orientation $6^{\circ}$ towards [110]. The source materials were trimethylgallium (TMGa), $100 \%$ arsine $\left(\mathrm{AsH}_{3}\right), 104-\mathrm{ppm}$ silane $\left(\mathrm{SiH}_{4}\right)$ as an $n$-type dopant, and palladium purified $\mathrm{H}_{2}$ as the carrier gas. During the growth, the pressure inside the reactor was kept at 100 Torr and the growth temperature was varied from 600 to $725^{\circ} \mathrm{C}$. The TMGa and $\mathrm{AsH}_{3}$ flow rates were varied from 5 to 20 SCCM and from 30 to 100 SCCM, respectively. The total flow rate was kept at about 2 standard liter per minute (SLPM). Prior to growth, the Ge substrates were degreased with organic solvents, then etched in 1:1:30 HF: $\mathrm{H}_{2} \mathrm{O}_{2}: \mathrm{H}_{2} \mathrm{O}$ for $15 \mathrm{~s}$. The details of the growth procedures can be found elsewhere. ${ }^{26-30}$

The double crystal x-ray diffraction (DCXRD) technique was used to measure the perpendicular lattice spacing $a_{\perp}$ of the epitaxial film and also the quality of the film. The epitaxial GaAs/Ge heterointerfaces were prepared by $\mathrm{Ar}^{+}$ion thinning for cross-sectional observations. Cross-sectional electron microscopy investigations were performed using a Hitachi H-9000 UHR ultrahigh resolution transmission electron microscope operated at $300 \mathrm{kV}$ to characterize the interface quality and the nature of the defects. PL measurements were carried out using a MIDAC Fourier transform PL (FTPL) system at a temperature of $4.2 \mathrm{~K}$ and $100 \mathrm{~mW}$ laser power for the determination of interface and surface roughness. The PL spectra were taken from the top surface and the close vicinity of the GaAs/Ge heterointerface for the observation of the self-annihilation of APBs.

\section{RESULTS AND DISCUSSION}

\section{A. Cross-sectional TEM observation of the $\mathrm{GaAs} / \mathrm{Ge}$ heterointerface}

The epitaxial films were investigated using TEM to reveal the characteristics of misfit dislocations (MDs) and other crystalline defects. In general, the dominant crystalline defects observed in the GaAs epifilm grown on Ge by MOVPE are APDs and dislocations (either misfit, threading, or both). Figure 1 shows the cross-sectional high-resolution TEM image of the GaAs/Ge heterointerface, grown using an As prelayer. From Fig. 1 it is seen that there are many threading dislocation groups and few APDs at the heteroint-

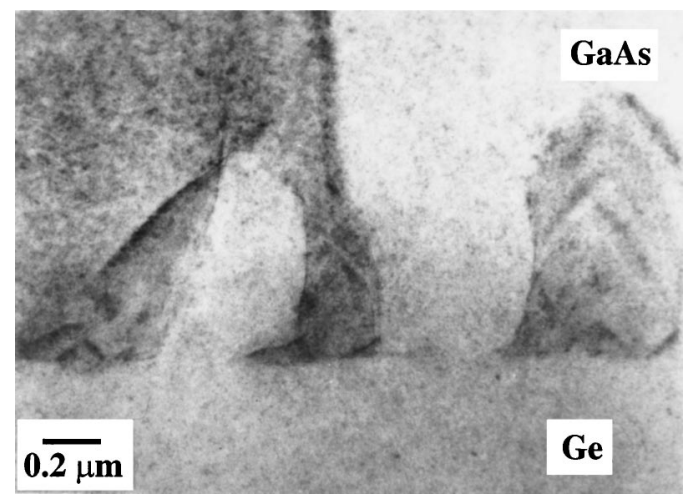

FIG. 2. [110] TEM cross-sectional micrograph of the GaAs/Ge heterointerface grown at $700{ }^{\circ} \mathrm{C}$ and a growth rate of $12 \mu \mathrm{m} / \mathrm{h}$ with a V/III ratio of 29.40 .

erface, and that they extended to the top surface of the GaAs film, similar to what has been observed by several authors. ${ }^{4,6,31-33}$ Ideally, APDs should not be observed in the presently investigated film due to the off-orientation of the substrate. ${ }^{2,4,34}$ Besides causing enhanced impurity diffusion, threading lines can also cause partial short circuiting of $p-n$ junctions and degrade the optical and electrical properties of the epilayer. ${ }^{35}$ Such threading lines can be overcome by introducing an additional strain field due to the presence of strained layer superlattices between the GaAs base and the Ge substrate. This strain field and the thread line will interact with each other during the epitaxial growth process, and the strain field in the epilayer can be used to bend the dislocation line. ${ }^{35-37}$ One approach is to bend the thread line towards another thread so that they annihilate each other. Another approach is to bend the thread line away from the surface towards the edge and parallel to the growth plane so that the thread line does not reach the surface at all.

Figure 2 shows a TEM cross-sectional micrograph of a $\mathrm{GaAs} / \mathrm{Ge}$ heterointerface grown at $700{ }^{\circ} \mathrm{C}$ using a V/III ratio of 29.40 and a growth rate of $12 \mu \mathrm{m} / \mathrm{h}$. From Fig. 2 it is seen that the interface between the GaAs epilayer and the Ge substrate is not sharp and in addition to MDs, threading dislocations and APDs are observed, which has been reported by several authors. ${ }^{18,34}$ Defects that propagate from the interface to the top of the layer likely originate during the early stages of the epitaxial growth. Generally, the MDs are seen at the heterointerface if the film thickness is greater than the critical

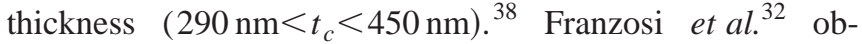
served that the epilayer crystal quality is strongly affected by the growth rate; layers grown at low growth rate $(1 \mu \mathrm{m} / \mathrm{h})$ exhibit planar defects, which are not found to be present in films grown at high deposition rates $(10 \mu \mathrm{m} / \mathrm{h})$. It has been recently argued that lattice mismatch plays only a minor role in the formation of planar defects such as twins and stacking faults. ${ }^{39,40}$

Figure 3 shows a TEM cross-sectional image of GaAs on Ge grown at a V/III ratio of 88.20 , using a growth rate of $6 \mu \mathrm{m} / \mathrm{h}$ and a growth temperature of $700^{\circ} \mathrm{C}$. It was found from this TEM photograph that the APB density decreases with distance from the GaAs/Ge interface. The reason is that the starting part of the APBs is inclined. Hence the probabil- 


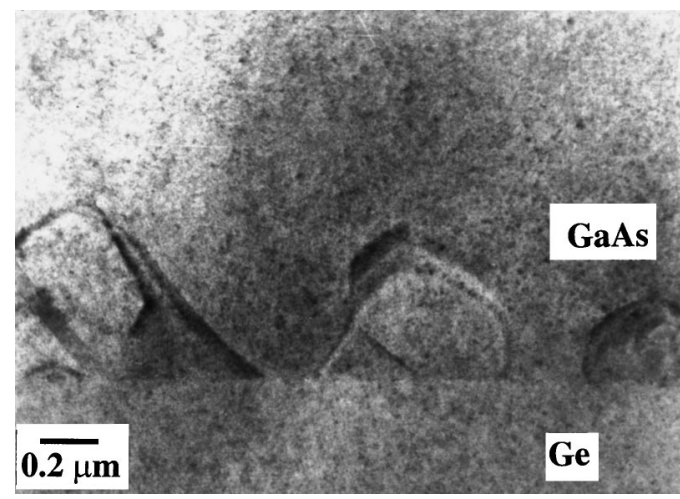

FIG. 3. [110] TEM cross-sectional micrograph of the GaAs/Ge heterointerface grown at $700{ }^{\circ} \mathrm{C}$ and a growth rate of $6 \mu \mathrm{m} / \mathrm{h}$ with a V/III ratio of 88.20 .

ity that two adjacent inclined APBs tend towards each other is high, and they self-annihilate. Thus most of the APDs disappear within a short distance from the interface. It can also be seen from this figure that for the layer grown under these conditions, the defects (mainly APDs) are confined to within $130 \mathrm{~nm}$ within the GaAs/Ge interface, and they do not propagate to the top surface of the film. The annihilation of APBs that have not been paired off near the interface is less probable because (i) they are no longer in close proximity to each other and (ii) they straighten up as they grow away from the interface. They become vertical beyond $130 \mathrm{~nm}$ from the interface (Fig. 3). Similar observations have been made by Guelton et al. ${ }^{16}$ in the GaAs/Ge system grown by close-spaced vapor transport, by Lazzarini et al. ${ }^{13}$ in GaAs/Ge grown by MOVPE, and by Koh et al. ${ }^{17}$ in GaAs/ $\mathrm{Ge} / \mathrm{GaAs}$ grown by MBE. The APBs annihilate each other during the thick GaAs epitaxial layer growth. The emergence of APBs gives rise to typical surfaces whose roughness depends on the density of emerging APBs. If the threading dislocations are oriented $60^{\circ}$ with respect to the substrate, it is very difficult to bend the thread towards the substrate. If the strain field is very high, then the APDs will not propagate to the top surface, but rather will remain confined to the near vicinity of the GaAs/Ge heterointerface. These dislocations are harmful and called type II dislocations; they can be converted into benign type I dislocations after proper annealing. ${ }^{35}$

Nucleation of GaAs directly on the Ge surface (without any epitaxial Ge growth) typically resulted in high defect densities in the GaAs epilayer due to the uncontrolled initial surface. ${ }^{41}$ Ringel et al. ${ }^{41}$ have found that a Ge epitaxial film annealed above $640^{\circ} \mathrm{C}$ for $\sim 20 \mathrm{~min}$, coupled with a large $6^{\circ}$ off-cut, results in double stepped Ge surfaces, which greatly suppressed APD formation. ${ }^{34}$ They also pointed out that the growth on Ge surfaces, which were not sufficiently annealed, typically showed high APD densities. The substrate temperature during the initial $100 \mathrm{~nm} \mathrm{GaAs}$ growth was very critical. Growth at too low a temperature resulted in an excess of As point defects, which enhance the nucleation loops. These loops expanded during the subsequent high temperature GaAs growth, which generated high threading dislocation densities in the thick GaAs film. ${ }^{31}$ On the other hand, growth

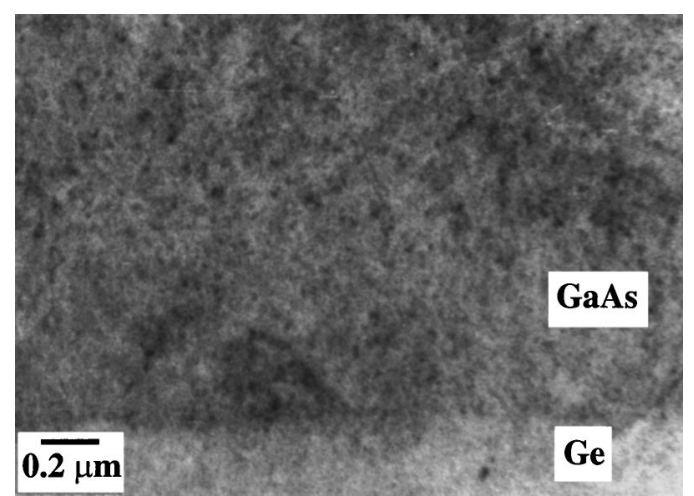

FIG. 4. [110] TEM cross-sectional micrograph of the GaAs/Ge heterointerface grown at $675^{\circ} \mathrm{C}$ and a growth rate of $3 \mu \mathrm{m} / \mathrm{h}$ with a V/III ratio of 88.20.

at higher temperatures and low growth rates may result in the formation of an unwanted $p-n$ junction due to simultaneous indiffusion of $\mathrm{Ga}$ and As into the Ge substrate, which in turn reduces the solar cell efficiency. ${ }^{42}$ Chen et al. ${ }^{43}$ studied the effect of growth temperature on the surface morphology of GaAs epitaxial layers on $2^{\circ}$ and $6^{\circ}$ off-cut Ge (001) substrates. They pointed out that the surface morphology of the layers grown at higher temperature $\left(730^{\circ} \mathrm{C}\right)$ was rough regardless of what the initial layer growth temperature was. The transition of APD-free $\rightarrow$ APDs $\rightarrow$ APD-free film with increasing growth temperature has already been observed experimentally by Fischer et al. ${ }^{8}$ in MBE grown GaAs on $\mathrm{Si}$ substrates. Li et al. ${ }^{6}$ pointed out that such a transition temperature should depend on other parameters as well, such as the substrate misorientation angle and the growth rate in the case of MOVPE growth of GaAs on Ge substrates. We also believe that the initial growth temperature, growth rate, and the substrate off-orientation play an important role in achieving APD-free GaAs on Ge substrates using the LPMOVPE growth process. ${ }^{26,44,45}$

Figure 4 shows a cross-sectional TEM image of a sample grown at $675^{\circ} \mathrm{C}$ using a V/III ratio of 88.20 and a growth rate of $3 \mu \mathrm{m} / \mathrm{h}$. It can be seen from this figure that the $\mathrm{GaAs} / \mathrm{Ge}$ interface is extremely abrupt, apart from the misfit dislocations. There are no threading dislocations or APBs present inside the film. This is due to the annihilation of the APBs, and thus the proper selection of the growth parameters. The selective area electron diffraction pattern (SAED) from the film and the substrate is shown in Fig. 5. It consists of strong spots, which indicates that both the film and the substrate are single crystalline.

\section{B. Observation of surface morphology by SEM studies}

The annihilation of APBs during epitaxial growth is shown clearly by observing the surface morphology by SEM after depositing a thick $3 \mu \mathrm{m}$ GaAs epitaxial layer. Figure 6 shows the surface morphology of a GaAs epitaxial layer on a Ge substrate. It is seen from Fig. 6 that the surface is wavy and that the APDs, which appear on the initial surface, are completely suppressed during the growth of the thick GaAs layer, this is similar to what is described by Li and Giling. ${ }^{12}$ 

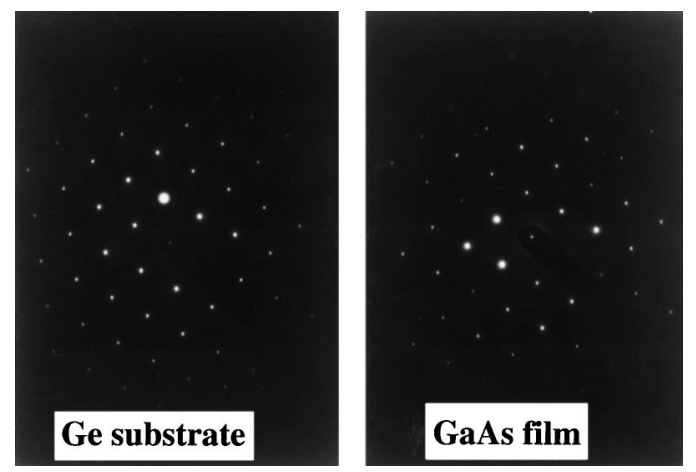

FIG. 5. Selective area electron pattern from (a) epi-GaAs film and (b) (100) Ge substrate.

The self-annihilation of APBs occurs by the rearrangement of randomly oriented APBs into ordered APBs, which lie in $\{011\}$ planes, followed by the propagation of APBs along the $\{011\}$ planes [which have an angle of $45^{\circ}$ with the $(001)$ surface] until they completely annihilate at their intersections. The initial APD density at the GaAs/Ge heterointerface can be expected to increase as the substrate offorientation angle is increased. Thus self-annihilation of APBs near the GaAs/Ge interface should occur at high offcut as a result of the increased probability that neighboring APBs will find one another and form small closed domains.

In the limit of vicinal (001) substrates, however, the spacing between adjacent APDs is expected to be quite large, so that self-annihilation near the interface and thus single domain growth become far less likely. ${ }^{31}$ The film surface was rough due to the presence of APBs inside the GaAs epitaxial layer as determined by atomic force microscopy (AFM), and $\mathrm{Xu}$ et al $^{46}$ have made a similar observation. The films grown at high temperatures showed a large number of APBs, and the surfaces were rough. ${ }^{45}$ The initial temperature is very critical for the MOVPE growth of APD-free GaAs on Ge substrates. Either the film surface or the close vicinity of the GaAs/Ge heterointerface will be rough, depending on the initial temperature of the GaAs growth. This can be confirmed after observing the PL spectra originating from the top surface and the immediate vicinity of the GaAs/Ge heterointerface. Li and Giling ${ }^{12}$ studied the self-annihilation of APBs after observing differential interference contrast mi-

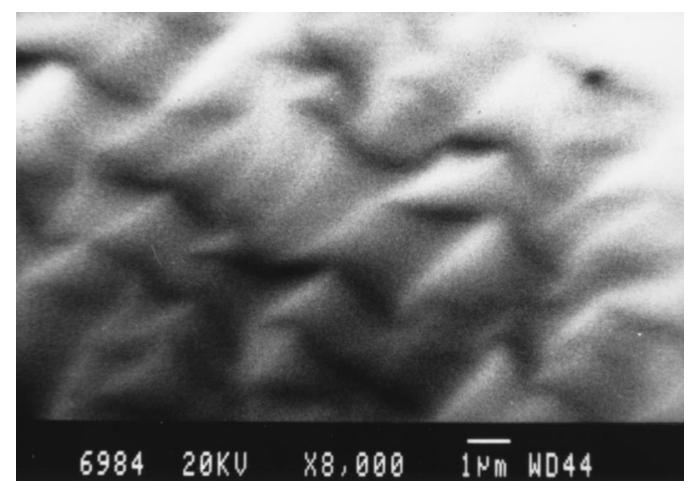

FIG. 6. SEM micrograph of a typical GaAs surface on Ge substrate. SEM micrograph demonstrating the self-annihilation of APBs.
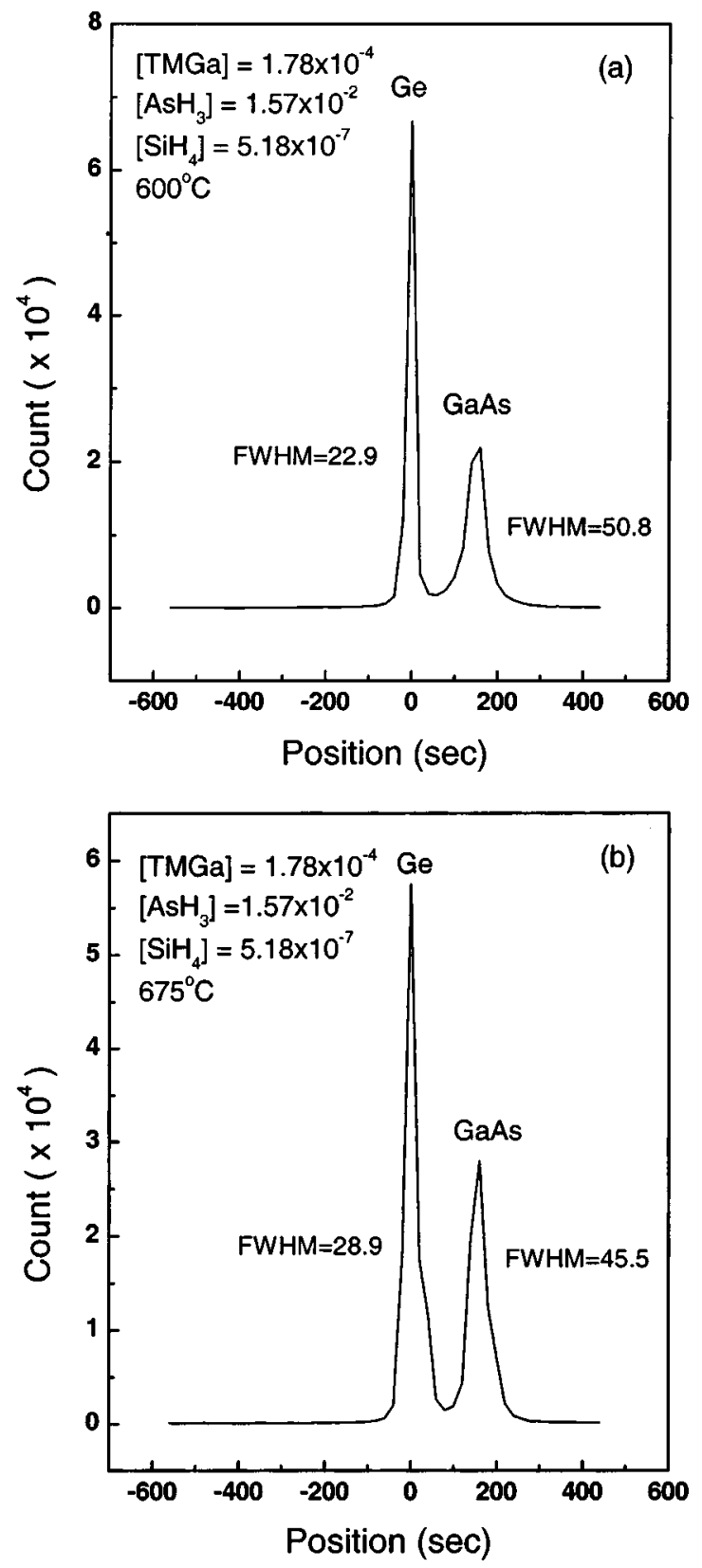

FIG. 7. DCXRD rocking curves from the (400) Bragg lines of GaAs epitaxial films grown at two different temperatures (a) $600{ }^{\circ} \mathrm{C}$ and (b) $675^{\circ} \mathrm{C}$ on Ge substrates.

croscopy (DICM) micrographs of both the as-grown GaAs film as well as micrographs from deeper in the film. The latter was achieved by sequential etching plus mechanochemical polishing of the GaAs on Ge (100). They found that the APBs annihilate each other after growing of the 4 $\mu \mathrm{m}$ thick GaAs layer.

\section{Double crystal x-ray diffraction study of GaAs layers}

\section{Effect of growth temperature on x-ray rocking curves}

$\mathrm{X}$-ray rocking curves of undoped and $\mathrm{Si}$-doped $\mathrm{GaAs} / \mathrm{Ge}$ epitaxial layers were examined using DCXRD. The $\mathrm{Cu} K \alpha_{1}$ radiation was used as a source of X-rays. Figures 7(a) and 
7(b) show typical DCXRD rocking curves from the (400) Bragg lines of GaAs epitaxial films grown at two different temperatures on $\mathrm{Ge}$ substrates. In both cases, the epitaxial layer thickness is larger than the critical layer thickness. The average separation between the Ge substrate peak and the GaAs epitaxial layer peak in these two cases is about 220 and 200-220 s. The angular separation $\Delta \theta$ between the (400) diffraction peaks of GaAs and Ge resulting from the difference in lattice plane spacing $\Delta d / d$, along with their diffraction line profiles, provided information about the microstructural quality of the GaAs films. The location of the peak associated with the epitaxial layer relative to the substrate, in our case, indicates that the Bragg angle of the epitaxial layer has increased and therefore the lattice is contracted. From the figure, one can see that the full width at half maximum (FWHM) of the GaAs epitaxial film peak decreases with increasing growth temperature and this confirms the selfannihilation of APBs. The FWHM of the Ge substrate peak affects with the increasing growth temperature. The narrowness of the FWHM of the GaAs epitaxial film peak indicates that the microstructural quality of the film is good.

\section{Effect of Si doping on x-ray rocking curves}

Figures 8(a) and 8(b) show the DCXRD rocking curve of GaAs epitaxial films grown at $650{ }^{\circ} \mathrm{C}$ on $\mathrm{Ge}$ substrates at different $\mathrm{SiH}_{4}$ mole fractions. From this figure, it can be seen that the FWHM of the GaAs epitaxial film increases due to the Si doping and that this also affects the FWHM of the Ge substrate, although the film is grown at a relatively low growth temperature. The Si doping in the GaAs on the Ge substrate may create strain that could affect the film properties. However, this is only possible in the Si-doping concentration range of $10^{18}$ to $10^{19} \mathrm{~cm}^{-3}$. ${ }^{47}$ Therefore the strain (assumed to be generated by growth temperature only) reduces or increases the interface roughness, due to the annihilation or generation of APBs, depending on the initial growth temperatures. Films grown at either $700{ }^{\circ} \mathrm{C}$ or higher show rough surfaces ${ }^{45}$ as observed by AFM; this may be due to APBs propagating from the near vicinity of the GaAs/Ge heterointerface to the top surface of the film during the growth process. Therefore the annihilation or the generation of the APBs with growth temperature is an important issue. This may be further confirmed by observing the optical properties of the films near the $\mathrm{GaAs} / \mathrm{Ge}$ heterointerface and the top surface of the GaAs epitaxial films. Table I shows the FWHM of the GaAs epitaxial layers on Ge substrates, and the surface and the interface roughness correlated with growth temperature.

\section{Low temperature photoluminescence studies}

Prior to the growth of Si-doped GaAs on Ge, undoped GaAs on $\mathrm{Ge}(\sim 2 \mu \mathrm{m})$ was grown in order to check the Ge outdiffusion into the film. Figure 9 shows one of the PL spectrums of an undoped GaAs epitaxial film on a Ge substrate. From this figure it is seen that the film has only one peak at $1.5125 \mathrm{eV}$ corresponding to the acceptor bound exciton; it has a FWHM of $10.3 \mathrm{meV}$. This PL spectrum suggests that $\mathrm{Ge}$ outdiffusion from the substrate is negligible. If
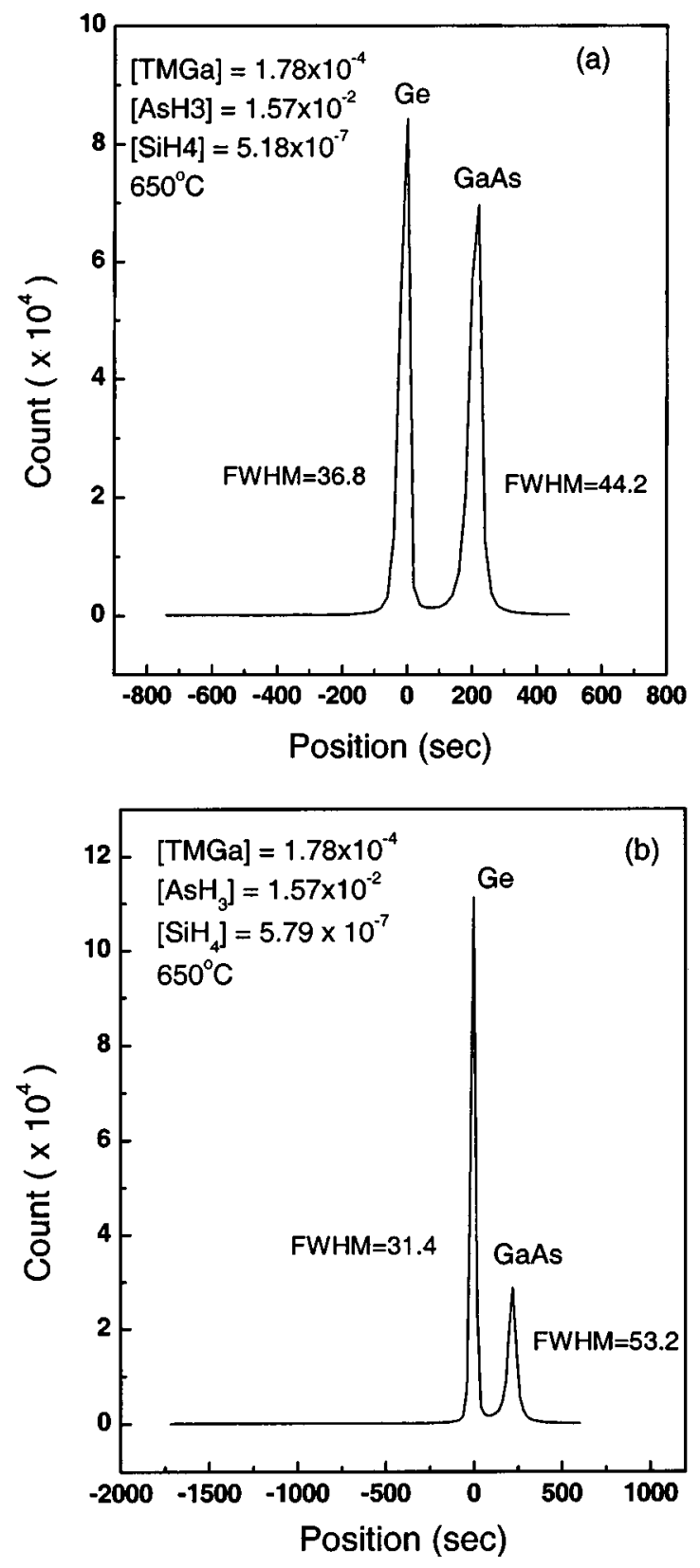

FIG. 8. DCXRD rocking curves from the (400) Bragg lines of GaAs epitaxial films grown at two different $\mathrm{SiH}_{4}$ mole fractions (a) $5.18 \times 10^{-7}$ and (b) $5.79 \times 10^{-7}$ on Ge substrates.

Ge outdiffusion (the Ge binding energy in GaAs is $43 \mathrm{meV}$ ) is present inside the GaAs epitaxial films, one should observe the peak at $1.474 \mathrm{eV}$ in a low temperature PL measurement. Since there is no peak at $1.474 \mathrm{eV}$ under our present growth conditions, we may assume that there is insignificant outdiffusion of Ge into the GaAs film and hence that the film is of high quality.

The above results confined the optical quality of the film near the surface. In order to further confirm insignificant outdiffusion of Ge from the substrate into the GaAs epitaxial film, PL spectra were taken after etching off about $1.3 \mu \mathrm{m}$ of the GaAs epitaxial layer using an electrochemical capacitance-voltage (ECV) profiler. A typical PL spectrum of the GaAs epitaxial layer after etching is shown in Fig. 9. From this figure it is seen that the peak appears at $1.516 \mathrm{eV}$, 
TABLE I. The FWHM, surface and interface roughness with the growth temperatures of GaAs epilayer on Ge substrate. The $\uparrow$ indicate increases and $\downarrow$ indicates decreases.

\begin{tabular}{cccc}
\hline \hline \multicolumn{5}{c}{ DCXRD results } \\
\hline Growth temperature $\left({ }^{\circ} \mathrm{C}\right)$ & FWHM of GaAs layer $(\mathrm{s})$ & {$\left[\mathrm{SiH}_{4}\right]$} \\
\hline 600 & & 50.8 & $5.18 \times 10^{-7}$ \\
625 & 50.5 & $5.18 \times 10^{-7}$ \\
650 & & 47.3 & $5.18 \times 10^{-7}$ \\
675 & \multicolumn{4}{c}{ LTPL results } & $5.18 \times 10^{-7}$ \\
& & & \\
\multicolumn{5}{c}{ Growth } & Surface & Interface & $\mathrm{SiH}_{4}$ \\
temperature $\left({ }^{\circ} \mathrm{C}\right)$ & roughness & roughness & mole fraction \\
\hline 625 & $\downarrow$ & $\uparrow$ & \\
625 & $\downarrow$ & $\downarrow$ & $5.18 \times 10^{-7}$ \\
650 & $\uparrow$ & $\downarrow$ & $5.18 \times 10^{-7}$ \\
700 & $\uparrow$ & $\downarrow$ & $5.18 \times 10^{-7}$ \\
\hline \hline
\end{tabular}

which corresponds to a free exciton in the GaAs epitaxial film, and confirms that the film is of high quality. Before etching the film, the PL peak was found at $1.5125 \mathrm{eV}$, which is an exciton bound-to-acceptor. The peak at $1.474 \mathrm{eV}$ was not observed even at a distance greater than $1.3 \mu \mathrm{m}$ below the top surface. The small shift in the peak $(1.5125 \mathrm{eV})$ may be attributed to possible Ge outdiffusion into the immediate vicinity of the GaAs film. This results in an increase in carrier concentration and a shift in the PL peak.

The PL spectra of Si-doped GaAs epitaxial films on Ge substrates before and after etching are shown in Fig. 10. It can be seen from this figure that under the process conditions specified in the figure, we did not observe any significant $\mathrm{Ge}$ outdiffusion from the substrate into the epilayer. If Ge outdiffuses into the epilayer, the electron concentration should

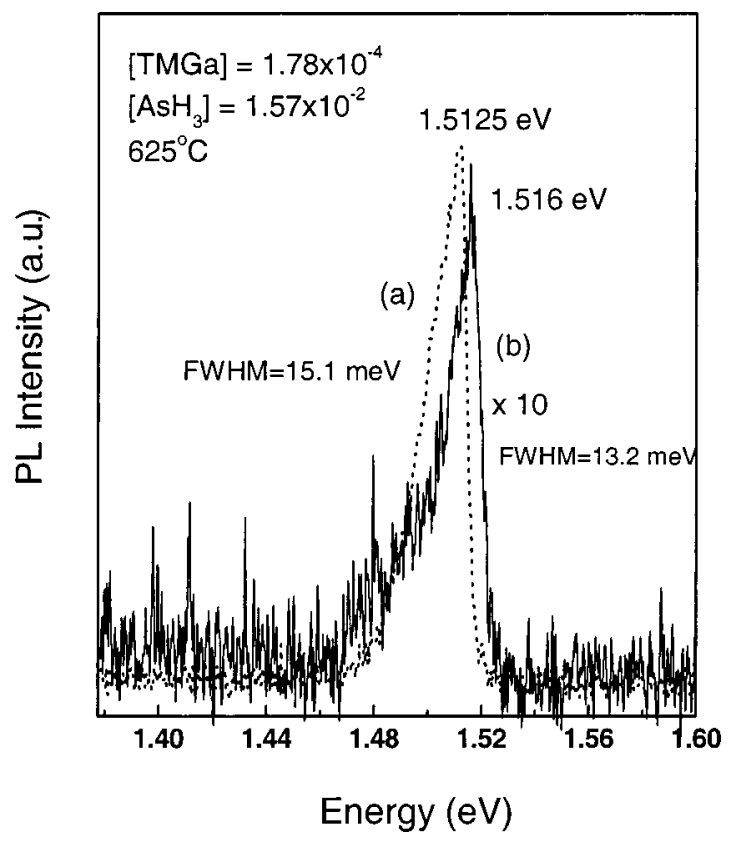

FIG. 9. 4.2 K PL spectra of undoped GaAs on Ge (a) at the surface (dotted line) and (b) at a depth of $1.3 \mu \mathrm{m}$ (solid surface) from the top surface. The thickness of the films was $2 \mu \mathrm{m}$.

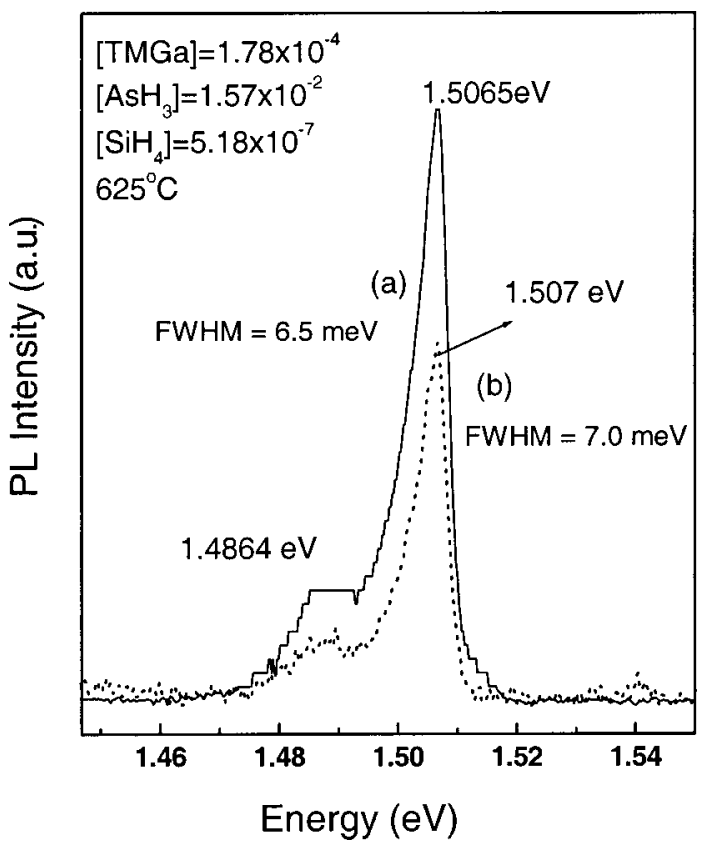

FIG. 10. 4.2 K PL spectra of Si-doped GaAs on Ge (a) at the surface (solid surface) and (b) at a depth of $0.8 \mu \mathrm{m}$ (dotted line) from the top surface. The thickness of the films was $1 \mu \mathrm{m}$.

increase due to the fact that Ge is an $n$-type dopant in $\mathrm{GaAs}^{28}$ under the epitaxial growth conditions used in these experiments. The increase in electron concentration leads to a shift in the PL main peak towards a higher energy, due to the Burstein-Moss effect. ${ }^{48}$ However, the outdiffusion of Ge of about $230 \mathrm{~nm}$ inside the GaAs epitaxial film $^{28}$ in the close vicinity of the GaAs/Ge heterointerface was noticed by secondary ion mass spectroscopy (SIMS). The peak at 1.4864 $\mathrm{eV}$ is due to the two-hole transition of an exciton bound to neutral Si donors. ${ }^{49}$

We have seen from the literature and also from our work $^{45}$ that the films grown at higher temperatures show a large number of APBs, which propagate to the top surface of the films. Figures 11 and 12 show the PL spectra of the films grown at higher temperatures; (a) and (b) indicate on both the figures obtained from the top show spectra obtained from the top surface and the close vicinity of the GaAs/Ge heterointerface, respectively. It can be seen from both of the figures that the top surfaces of both of the films were very rough due to the higher noise level present, and that the intensity of the PL peak was lower as compared with the intensity from the interface region. This clearly indicates the propagation of APBs to the top surface of the films, which makes the film surface appear very rough. On the other hand, one can see from Figs. 9 and 10 that the PL spectrum taken below the surface shows more noise as compared to the top surface spectrum, and also that the PL peak intensity was much less compared to that taken at the top surface. This analysis tells us that near the heterointerface $(\approx 0.1 \mu \mathrm{m}$ from the Ge surface) the GaAs film was rough when compared to the top surface. This may be due to a higher density of APBs within the first few $\mathrm{nm}$ of the epilayer, which annihilate each other after the growth of a thick GaAs layer. This has been previously described by Li and coworkers. ${ }^{5,6,12}$ Therefore we can 


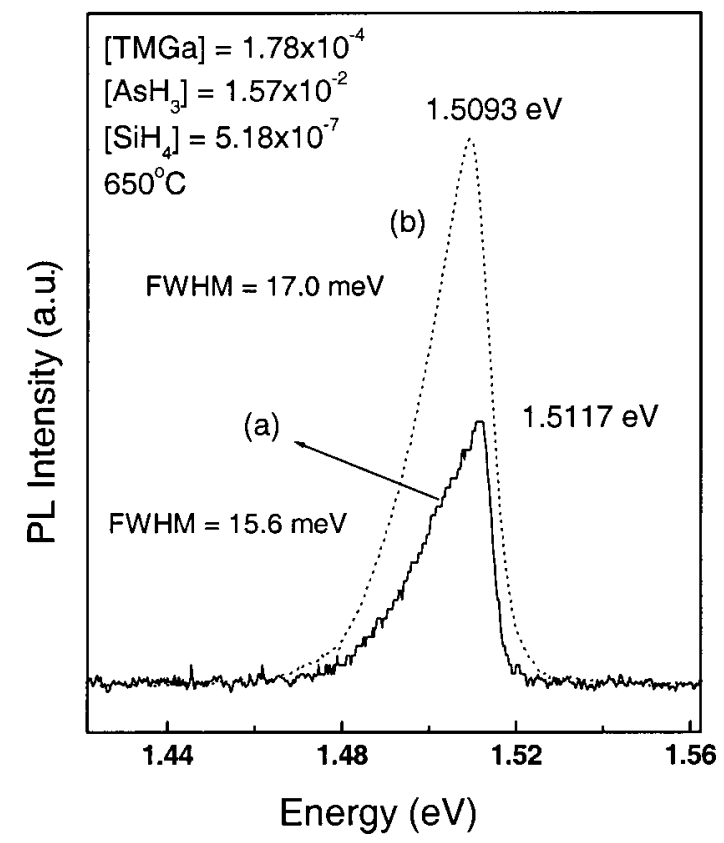

FIG. 11. 4.2 K PL spectra of Si-doped GaAs on Ge (a) at the surface (solid surface) and (b) at a depth of $0.8 \mu \mathrm{m}$ (dotted line) from the top surface. The thickness of the films was $1 \mu \mathrm{m}$ and the growth temperature was $650{ }^{\circ} \mathrm{C}$.

conclude that the initial temperature of the MOVPE growth of GaAs on Ge substrate is critical. More APBs appear near the interface at low growth temperatures, which annihilate at higher growth temperatures. Further, more APBs are present at the top surface at higher growth temperatures.

\section{CONCLUSIONS}

Undoped and Si-doped GaAs epitaxial layers were grown by the LPMOVPE growth on $6^{\circ} \mathrm{Ge}$ substrates tech-

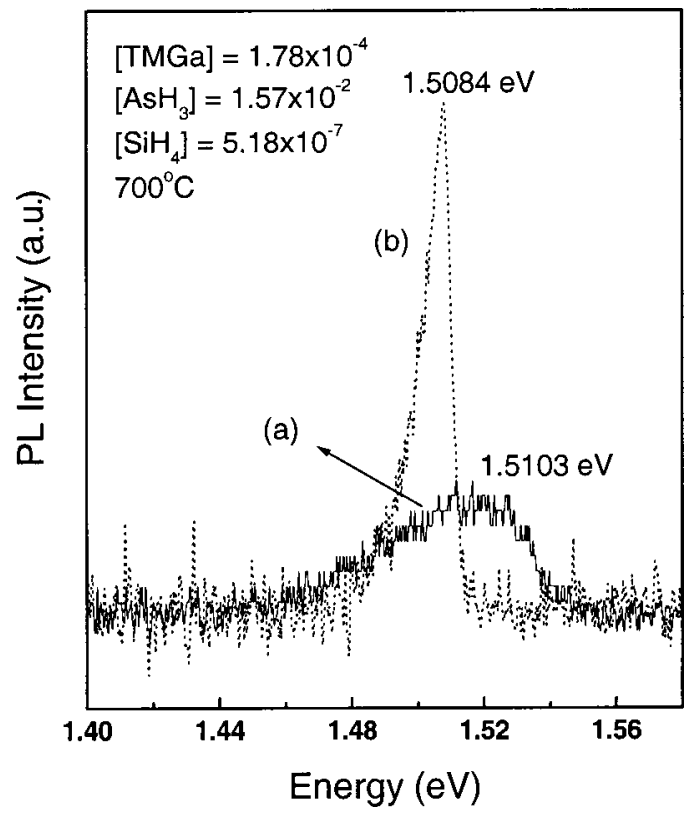

FIG. 12. 4.2 K PL spectra of Si-doped GaAs on Ge (a) at the surface (solid surface) and (b) at a depth of $0.8 \mu \mathrm{m}$ (dotted line) from the top surface. The thickness of the films was $1 \mu \mathrm{m}$ and the growth temperature was $700{ }^{\circ} \mathrm{C}$. nique and were studied by several characterization techniques. Cross-sectional TEM studies showed that APD-free GaAs growth on $\mathrm{Ge}$ was possible if the growth parameters were properly selected. The APBs annihilate each other after a thick layer of GaAs growth on Ge substrates, as observed by SEM. DCXRD data showed slightly compressed GaAs on $\mathrm{Ge}$, and the FWHM of the GaAs epilayer decreases with increasing growth temperatures. This confirms that the APBs annihilate inside the GaAs epitaxial films. LTPL measurements further confirmed the self-annihilation of the APBs in the low temperature growth regime and the generation of APBs at higher growth temperatures.

${ }^{1}$ H. Kroemer, J. Cryst. Growth 81, 193 (1987).

${ }^{2}$ P. R. Pukite and P. I. Cohen, J. Cryst. Growth 81, 214 (1987).

${ }^{3}$ P. M. Petroff, J. Vac. Sci. Technol. B 4, 874 (1986).

${ }^{4}$ K. Mizuguchi, N. Hayafuji, S. Ochi, T. Murotani, and K. Fujikawa, J. Cryst. Growth 77, 509 (1986).

${ }^{5}$ Y. Li, L. Lazzarini, L. J. Giling, and G. Salviati, J. Appl. Phys. 76, 5748 (1994).

${ }^{6}$ Y. Li, G. Salviati, M. M. G. Bongers, L. Lazzarini, L. Nasi, and L. J. Giling, J. Cryst. Growth 163, 195 (1996).

${ }^{7}$ M. Kawabe and T. Udeda, Jpn. J. Appl. Phys., Part 2 26, L944 (1997).

${ }^{8}$ R. Fischer, H. Morkoç, D. A. Neumann, H. Zabel, C. Choi, N. Otsuku, M. Longerbone, and L. P. Erichson, J. Appl. Phys. 60, 1640 (1986).

${ }^{9}$ S. Strite, D. Biswas, N. S. Kumar, N. Fradin, and H. Morkoç, Appl. Phys. Lett. 56, 244 (1990)

${ }^{10}$ L. Lazzarini, Y. Li, P. Franzosi, L. J. Giling, L. Nasi, F. Longo, M. Urchulutegui, and G. Salviati, Mater. Sci. Eng., B 28, 502 (1994).

${ }^{11}$ S. I. Molina, G. Aragoni, R. Garcia, Y. Gonzalez, and F. Briones, J. Electron. Mater. 22, 567 (1993).

${ }^{12}$ Y. Li and L. J. Giling, J. Cryst. Growth 163, 203 (1996).

${ }^{13}$ L. Lazzarini, L. Nasi, G. Salviati, C. Z. Fregonara, Y. Li, L. J. Giling, C. Hardingham, and D. B. Holt, Micron 31, 217 (2000).

${ }^{14}$ A. Georgakilas, J. Stoemenos, K. Tsagaraki, P. Komninou, N. Flevaris, P. Panyotatos, and A. Christou, J. Mater. Res. 8, 1908 (1993).

${ }^{15}$ M. Yamaguchi, J. Mater. Res. 6, 376 (1991).

${ }^{16}$ N. Guelton, R. G. Saint-Jacques, G. Lalande, and J-P. Dodelet, J. Mater. Res. 10, 843 (1995)

${ }^{17}$ S. Koh, T. Kondo, T. Ishiwada, H. Sawada, H. Ichinose, I. Shoji, and R. Ito, Physica E (Amsterdam) 7, 876 (2000).

${ }^{18}$ H. Kroemer, J. Vac. Sci. Technol. 5, 1150 (1987).

${ }^{19}$ E. P. Kvam, J. Electron. Mater. 23, 1021 (1994).

${ }^{20}$ S. Strite, D. Biswas, N. S. Kumar, M. Fradkin, and H. Morkoç, J. Appl. Phys. 67, 1609 (1990).

${ }^{21}$ N. Chand, J. Klem, T. Henderson, and H. Morkoç, J. Appl. Phys. 59, 3601 (1986).

${ }^{22}$ D. B. Holt, J. Phys. Chem. Solids 30, 1297 (1969).

${ }^{23}$ D. B. Holt, C. Hardingham, L. Lazzarini, L. Nasi, C. Z. Fregonara, G. Salviati, and M. Mazzer, Mater. Sci. Eng., B 42, 204 (1996).

${ }^{24}$ E. A. Fitzgerald, J. M. Kuo, Y. H. Xie, and P. J. Silverman, Appl. Phys. Lett. 64, 733 (1994)

${ }^{25}$ N. A. El-Masry, J. C. Tarn, and N. H. Karam, J. Appl. Phys. 64, 3672 (1988)

${ }^{26}$ M. K. Hudait and S. B. Krupanidhi, Mat. Sci. Eng., B (unpublished).

${ }^{27}$ M. K. Hudait, P. Modak, S. Hardikar, and S. B. Krupanidhi, J. Appl. Phys. 83, 4454 (1998).

${ }^{28}$ M. K. Hudait and S. B. Krupanidhi, J. Vac. Sci. Technol. B 17, 1003 (1999).

${ }^{29}$ P. Modak, M. K. Hudait, S. Hardikar, and S. B. Krupanidhi, J. Cryst. Growth 193, 501 (1998).

${ }^{30}$ M. K. Hudait, P. Modak, S. Hardikar, K. S. R. K. Rao, and S. B. Krupanidhi, Mater. Sci. Eng., B 55, 53 (1998).

${ }^{31}$ S. M. Ting, E. A. Fitzgerald, R. M. Sieg, and S. A. Ringel, J. Electron. Mater. 27, 451 (1998)

${ }^{32}$ P. Franzosi, L. Lazzarini, G. Salviati, M. Scafferdi, and G. Timò, Inst. Phys. Conf. Ser. 117, 399 (1991).

${ }^{33}$ J. M. Kuo, E. A. Fitzgerald, Y. H. Xie, and P. J. Silverman, J. Vac. Sci. Technol. B 11, 857 (1993).

${ }^{34}$ S. Strite, M. S. Unlu, K. Adomi, G. B. Gao, A. Agarwal, A. Rockett, H. 
Morkoç, D. Li, Y. Nakamura, and N. Otsuka, J. Vac. Sci. Technol. B 8, 1131 (1990).

${ }^{35}$ S. F. Fang, K. Adomi, S. Iyer, H. Morkoç, H. Zabel, C. Choi, and N. Otsuka, J. Appl. Phys. 68, R31 (1990).

${ }^{36}$ J. W. Mattews and A. E. Blakeslee, J. Cryst. Growth 29, 273 (1975).

${ }^{37}$ J. W. Mattews and A. E. Blakeslee, J. Cryst. Growth 32, 265 (1976).

${ }^{38}$ G. Timò, C. Flores, R. Campesato, D. Passoni, and B. Bollani, Mater. Sci. Forum 203, 97 (1996)

${ }^{39}$ J. W. Lee and H. L. Tsoi, J. Vac. Sci. Technol. B 5, 819 (1987).

${ }^{40}$ G. Timò, C. Flores, B. Bollani, D. Passoni, C. Bocchi, P. Franzosi, L. Lazzarini, and G. Salviati, J. Cryst. Growth 125, 440 (1992).

${ }^{41}$ S. A. Ringel, R. M. Sieg, S. M. Ting, and E. A. Fitzgerald, Proceedings of the Twenty-sixth IEEE Photovoltaic Spec. Conference (IEEE, New York, 1997), p. 793.
${ }^{42}$ P. A. Iles, Y. C. M. Yeh, F. H. Ho, C. L. Chu, and C. Cheng, IEEE Electron Device Lett. EDL-11, 140 (1990).

${ }^{43}$ J. C. Chen, M. L. Ristow, J. I. Cubbage, and J. G. Werthen, J. Electron. Mater. 21, 347 (1992).

${ }^{44}$ M. K. Hudait and S. B. Krupanidhi, Mater. Res. Bull. 35, 125 (2000).

${ }^{45}$ M. K. Hudait and S. B. Krupanidhi, Mater. Res. Bull. 35, 909 (2000).

${ }^{46}$ Q. Xu, J. W. P. Hsu, S. M. Ting, E. A. Fitzgerald, R. M. Sieg, and S. A. Ringel, J. Electron. Mater. 27, 1010 (1998).

${ }^{47}$ R. Venkatasubramanian, K. Patel, and S. K. Ghandhi, J. Cryst. Growth 94, 34 (1989).

${ }^{48}$ E. Burstein, Phys. Rev. 93, 632 (1954); T. S. Moss, Proc. Phys. Soc. London, Sect. B 67, 775 (1954).

${ }^{49}$ L. Pavesi and M. Guzzi, J. Appl. Phys. 75, 4779 (1994). 